\title{
ON A THEOREM OF OSCILLATION.
}

BY PROFESSOR WILLIAM F. OSGOOD.

IN his book on series which represent the potential function Bôcher makes use of the first part of the following theorem.* All the variables are real.

THEOREM-Let $\varphi(t)$ be continuous and monotonic in the interval $T \leqq t<\infty$, and let $\varphi(t)$ be always greater numerically than a certain positive constant $\gamma$ :

$$
|\varphi(t)|>\gamma .
$$

If $\varphi(t)<0$, an arbitrary solution of the differential equation

$$
\frac{d^{2} y}{d t^{2}}=\varphi(t) y,
$$

oscillates an infinite number of times in any interval $T \leqq T^{\prime}$ $\leqq t<\infty$, in which it is considered, the amplitudes of the oscillations remaining finite.

If, furthermore, $\varphi(t)$ remains finite, the amplitudes of the oscillations do not become less than a certain positive constant. Moreover, the amplitudes vary monotonically, increasing when $\varphi(t)$ increases algebraically, and decreasing in the opposite case.

Bôcher states the theorem without the restriction that $\varphi(t)$ be monotonic, and outlines a suggestive dynamical proof. Professor Birkhoff's comment in the foregoing article led me to examine critically both theorem and proof. It is easy to see that the theorem is not true under Bôcher's hypotheses. He needed the theorem, however, only in the restricted form above given. Moreover, he does not state the last paragraph of the theorem, this extension not being requisite for his purposes.

As regards this extension, it is not difficult to show that, if $\varphi(t)$ is not required to remain finite, the amplitudes of the oscillations may become infinitesimal, when $t$ becomes infinite.

* Ueber die Reihenentwickelungen der Potentialtheorie; Teubner, 1894, p. 178

+ Cf. infra. 
Proof. Let

$$
p=p(t)=-\varphi(t), \quad p_{n}=p\left(t_{n}\right) .
$$

To integrate the differential equation

$$
\frac{d^{2} y}{d t^{2}}=-p y
$$

proceed in the usual manner:

$$
\begin{aligned}
2 \frac{d y}{d t} \frac{d^{2} y}{d t^{2}} & =-2 p y \frac{d y}{d t}, \\
\left(\frac{d y}{d t}\right)^{2} & =-2 \int p y d y+C .
\end{aligned}
$$

We will now introduce with Bôcher the dynamical interpretation of the equation (1) as representing Newton's second law of motion for a particle of unit mass acted on by a central attractive force whose intensity, in absolute units, is $p y$, and moving in a right line through the center of force $O$. Here, $t$ represents the time, and $y$ the distance, measured algebraically, of the particle from $O$.

We will determine the constant of integration for two particular cases.

Case A. The particle is projected from $O$ in the sense of the increasing $y$ with an initial velocity $v=c$. Then

$$
\left(\frac{d y}{d t}\right)^{2}=c^{2}-2 \int_{0}^{y} p y d y .
$$

The amplitude $h$ of this half-oscillation is given !by putting $d y / d t=0, y=h$. Hence

$$
\int_{0}^{h} p y d y=\frac{c^{2}}{2} .
$$

Case B. The particle is released from rest at the point $y=h>0$. Then

$$
\left(\frac{d y}{d t}\right)^{2}=2 \int_{y}^{h} p y d y .
$$

The velocity $c$ with which it reaches the center of force is given by the equation 


$$
c^{2}=2 \int_{0}^{h} p y d y
$$

which is of the same form as (2).

Thus both cases lead to the same formal relation (2) between $c$ and $h$.

Consider, now, an arbitrary solution $y$ of (1) in the interval $T \leqq t^{\prime} \leqq t<\infty$, and suppose

$$
\left.y\right|_{t=t^{\prime}}>0 \text {. }
$$

Since the force is attractive and its intensity is at least $\gamma y$, the particle will be pulled in to the center of force, and the given solution will vanish at a definite instant* $t=t_{0}>t^{\prime}$.

It is sufficient, then, to consider a half-oscillation, i. e., an excursion from the center of force back to that point. Let the particle be projected at the instant $t=t_{0}$ from $O$ with velocity $v_{0}$ in the direction of the positive $y$ 's.

I. Let the monotonic function $p(t)$ decrease. Then

$$
p(t)>\gamma, \quad p(\infty) \geqq \gamma .
$$

Corresponding to Case A, we have for the motion away from $O$ to rest

$$
\frac{p_{1} h_{1}^{2}}{2} \leqq \uparrow \int_{0}^{h_{1}} p y d y=\frac{v_{0}^{2}}{2}
$$

Hence

* The argument can be equally well carried through analytically. The curve corresponding to the solution in question, $y=f(t)$, is concave downward, since $d^{2} y / d t^{2}<0$. If $f^{\prime}\left(t^{\prime}\right)>0$, the curve must have a maximum and return to the initial level, $y=f\left(t^{\prime}\right)=b$. For, while it is above that level,

$$
d^{2} y / d t^{2}=-p y<-\gamma b=-k .
$$

Consider the parabola for which $d^{2} y / d t^{2}=-k$, and which is tangent initially to the above curve:

$$
Y=b+f^{\prime}\left(t^{\prime}\right)\left(t-t^{\prime}\right)-\frac{1}{2} k\left(t-t^{\prime}\right)^{2} .
$$

Then the given curve will lie below the parabola so long as it lies above the line $y=b$. For, by Taylor's theorem with the remainder,

Hence

$$
y=b+f^{\prime}\left(t^{\prime}\right)\left(t-t^{\prime}\right)+\frac{1}{2} f^{\prime \prime}(\tau)\left(t-t^{\prime}\right)^{2}, \quad t^{\prime}<\tau<t .
$$

$$
Y-y=\frac{1}{2}[p y-k]_{t=\tau}\left(t-t^{\prime}\right)^{2},
$$

and the bracket is positive so long as $y \geqq b$.

Thus the curve $y=f(t)$ cuts the line $\bar{y}=b$ a second time, and at this point the slope is negative. From now on the curve lies below its tangent at this point, till it cuts the axis of $t$. 


$$
h_{1} \leqq \frac{v_{0}}{\sqrt{p_{1}}} \leqq \frac{v_{0}}{\sqrt{\gamma}} .
$$

Corresponding to Case $\mathrm{B}$, we have for the motion back* to $O$

$$
\frac{p_{1} h_{1}^{2}}{2} \geqq \downarrow \int_{0}^{h_{1}} p y d y=\frac{v_{2}^{2}}{2} .
$$

From (3) and (4) we infer that

$$
v_{2} \leqq v_{0}
$$

It thus appears that, as the motion continues, the successive values $v_{0}, v_{2}, v_{4}, \cdots$ never increase, and hence $h_{n}$ remains finite, since

$$
h_{n+1} \leqq \frac{v_{2 n}}{\sqrt{\gamma}} \leqq \frac{v_{0}}{\sqrt{\gamma}}
$$

To show that $h_{n}$ remains greater than a certain positive constant, we replace (3) and (4) by the new relations

$$
\begin{aligned}
& \frac{p_{0} h_{1}^{2}}{2} \geqq \uparrow \int_{0}^{h_{1}} p y d y=\frac{v_{2}^{2}}{2} ; \\
& \frac{p_{2} h_{1}^{2}}{2} \leqq \downarrow \int_{0}^{h_{1}} p y d y=\frac{v_{2}^{2}}{2} .
\end{aligned}
$$

Hence,

$$
h_{1} \geqq \frac{v_{0}}{\sqrt{p_{0}}}, \quad \frac{v_{2}}{\sqrt{p_{2}}} \geqq \frac{v_{0}}{\sqrt{p_{0}}} .
$$

On writing down the corresponding relations for the further half-oscillations and taking $v_{2 m}$ always to mean the numerical value of $d y / d t$ at $O$,

we obtain

$$
\begin{gathered}
\frac{v_{4}}{\sqrt{p_{4}}} \geqq \frac{v_{2}}{\sqrt{p_{2}}}, \\
\ldots \ldots \ldots \ldots \ldots . \\
\frac{v_{2 n}}{\sqrt{p_{2 n}}} \geqq \frac{v_{2 n-2}}{\sqrt{p_{2 n-2}}},
\end{gathered}
$$

$$
h_{n+1} \geqq \frac{v_{2 n}}{\sqrt{p_{2 n}}} \geqq \frac{v_{0}}{\sqrt{p_{0}}},
$$

${ }^{*} p$, considered as a function of $y$, is multiple-valued; but in each of the cases $A$ and $B$ we are dealing only with a single-valued branch of the function. 
and thus the theorem is proven for the case of a decreasing function $p(t)$, the corresponding function $\varphi(t)$ increasing.

Finally, $h_{n}$ increases monotonically,

since from (5) and (6)

$$
h_{n+1} \geqq h_{n},
$$

$$
\frac{v_{0}}{\sqrt{p_{0}}} \leqq h_{1} \leqq \frac{v_{2}}{\sqrt{p_{2}}}
$$

etc.

II. When $p(t)$ is an increasing function, the proof is given in a similar manner. Instead of (3) and (4) we now have

$$
\begin{aligned}
& \frac{p_{0} h_{1}^{2}}{2} \leqq \quad \int_{0}^{h_{1}} p y d y=\frac{v_{0}^{2}}{2} \\
& \frac{p_{2} h_{1}^{2}}{2} \geqq \downarrow \int_{0}^{h_{1}} p y d y=\frac{v_{2}^{2}}{2} .
\end{aligned}
$$

Here, we have

$$
h_{1} \leqq \frac{v_{0}}{\sqrt{p_{0}}}, \quad \frac{\left\lceil v_{2}\right.}{\sqrt{p_{2}}} \leqq \frac{v_{0}}{\sqrt{p_{0}}} .
$$

Hence

and $h_{n}$ remains finite.

$$
h_{n+1} \leqq \frac{v_{2 n}}{\sqrt{p_{2 n}}} \leqq \frac{v_{0}}{\sqrt{p_{0}}},
$$

To show that $h_{n}$ does not fall below a positive constant, write

$$
\begin{aligned}
& \frac{p_{1} h_{1}^{2}}{2} \geqq \uparrow \int_{0}^{h_{1}} p y d y=\frac{v_{0}^{2}}{2} \\
& \frac{p_{1} h_{1}^{2}}{2} \leqq \downarrow \int_{0}^{h_{1}} p y d y=\frac{v_{2}^{2}}{2} .
\end{aligned}
$$

Hence

and thus

$$
h_{1} \geqq \frac{v_{0}}{\sqrt{p_{1}}} \geqq \frac{v_{0}}{\sqrt{\gamma}}, \quad v_{2} \geqq v_{0}
$$

etc.

$$
h_{n+1} \geqq \frac{v_{0}}{\sqrt{\gamma}} .
$$

From (7) and (8) it follows that

$$
\frac{v_{2}}{\sqrt{p_{2}}} \leqq h_{1} \leqq \frac{v_{0}}{\sqrt{p_{0}}},
$$


Hence

$$
h_{n+1} \leqq h_{n},
$$

and $h_{n}$ decreases monotonically.

Physical Interpretation.-A physical interpretation of the problem just discussed is given by the small oscillations of a simple pendulum, the cord of which, in Case I, is gradually lengthened, monotonically, remaining finite; while in Case II the cord is monotonically shortened, never becoming less than a positive fixed length.

The same physical picture is useful, too, for showing that the theorem, stated without restriction on $\varphi(t)$, is false. For the way a child swings higher and higher is to lengthen the equivalent simple pendulum on the downward arc, and suddenly to shorten it as the upward arc begins.

Bôcher's theorem $(b), 1$. c., namely that, when $\varphi(t)$ is continuous and

$$
0<g \leqq \varphi(t) \leqq G,
$$

there is one and only one solution which remains finite, and this vanishes at infinity, is correct without any further restriction on the function $\varphi$. An elementary analytic proof can be given by the aid of the law of the mean and Taylor's theorem with the remainder, carried through the term of the second order.

HARVARD UNIVERSITY,

Cambridge, Massachusetts, December 8, 1918.

\section{PROOF OF A PROPERTY OF THE NORM OF A CYCLOTOMIC INTEGER.}

BY MR. H. S. VANDIVER.

(Read before the American Mathematical Society April 27, 1918.)

KUMmer in his first proof of the general law of reciprocity between two ideals in a regular cyclotomic algebraic field gave a theorem* which forms a link in his chain of reasoning. Let

* Abhandlungen Berlin Academy, 1859, p. 119, formula (7). 